Unkontrolliertes Asthma

\title{
Anticholinergikum so effektiv wie lang wirksames Betamimetikum
}

Peters SP et al. Tiotropium bromide step-up therapy for adults with uncontrolled asthma. N Engl J Med. 2010;363:1715-1726

\section{Hintergrund und Fragestellung}

In den Asthmaleitlinien werden als primäre Alternative zur bronchodilatativen Langzeittherapie nicht Anticholinergika empfohlen, sondern Beta-2-Mimetika. Ziel dieser von den National Institutes of Health (NIH) unterstützten Studie war es, zu untersuchen, ob dieses Paradigma berechtigt ist.

\section{Methodik}

In dieser Crossover- und Triple-dummyStudie wurde bei Patienten, die mit dem inhalativen Kortikosteroid (ICS) Beclo-

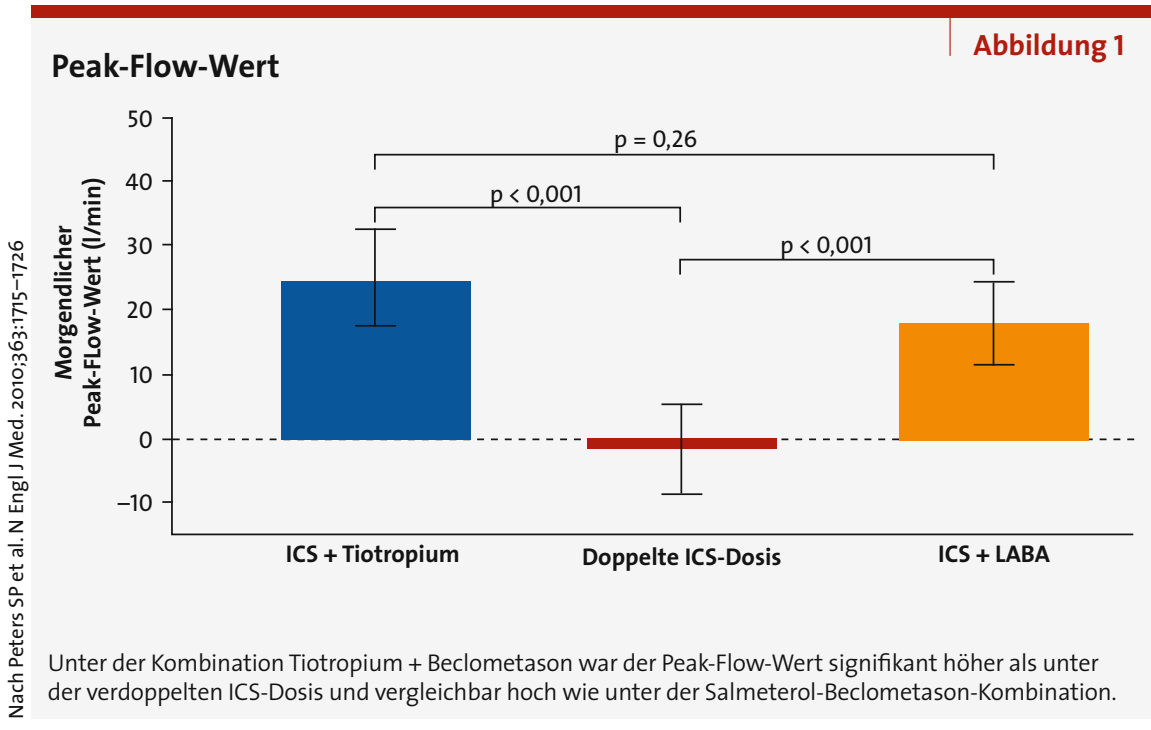

Einsekundenkapazität

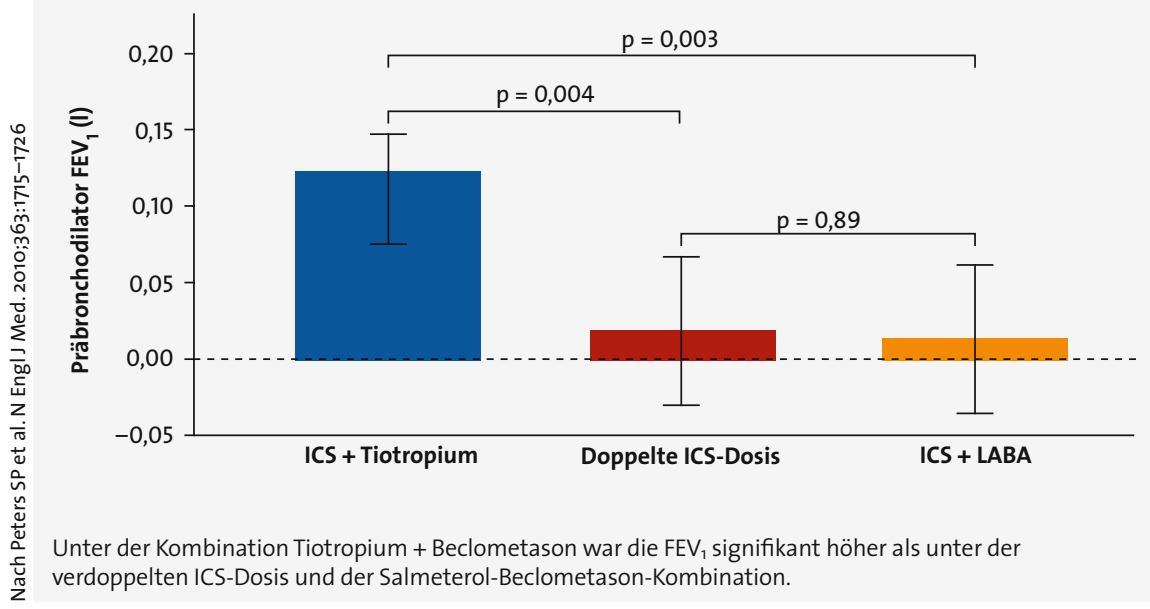

metason $(2 \times 80 \mu g)$ inadäquat eingestellt waren, untersucht, ob

— die zusätzliche Tiotropiumgabe der ICS-Monotherapie überlegen ist,

- die ICS-Tiotropium-Kombination einer Verdoppelung der ICS-Dosis überlegen ist und

- die ICS-Tiotropium-Kombination und eine Kombination aus einem lang wirksamen Beta-2-Mimetikum (LABA) und einem ICS gleichwertig sind.

210 Asthmapatienten mit einer durchschnittlichen Einsekundenkapazität $\left(\mathrm{FEV}_{1}\right)$ von $71,5 \%$ des Sollwerts wurden ausgewertet. Die Studiendauer pro Behandlungsarm betrug 14 Tage.

\section{Ergebnisse}

Die Tiotropium-ICS-Kombination verbesserte den Peak-Flow-Wert (primärer Endpunkt) vergleichbar gut wie die LABA-ICS-Kombination (Abb. 1). Hinsichtlich der $\mathrm{FEV}_{1}$ (sekundärer Endpunkt) war sie allen Vergleichsgruppen sogar überlegen (Abb. 2).

\section{Kommentar}

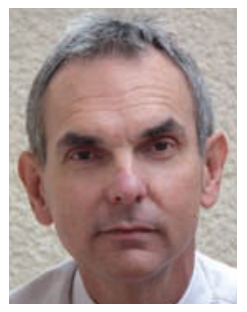

Prof. Dr. med.

Adrian Gillissen,

Kassel

Trotz der Kürze der Studiendauer von nur 14 Tagen war die Tiotropium-ICS-Kombination der Salmeterol-ICS-Kombination ebenbürtig (Peak-Flow-Wert) oder sogar überlegen $\left(\mathrm{FEV}_{1}\right)$.

Die in der Therapie von obstruktiven Atemwegserkrankungen Erfahrenen haben schon lange geahnt, dass Anticholinergika nicht nur bei der chronisch obstruktiven Lungenerkrankung eine gute bronchodilatative Wirkung besitzen. Ipratropium wird für die Notfalltherapie des Asthmas sogar ausdrücklich empfohlen.

Diese Studie zeigt in aller Deutlichkeit, dass es im Grunde nicht so wichtig ist, mit welchem Bronchodilatator ein ICS kombiniert wird, da jegliche ICS-BronchodilatatorKombination einer alleinigen ICS-Behandlung überlegen ist. 